Pure Appl. Chem., Vol. 72, No. 11, pp. 2083-2099, 2000.

(C) 2000 IUPAC

\title{
Temperature control modes in thermal analysis*
}

\author{
Takeo Ozawa ${ }^{\dagger}$
}

Chiba Institute of Technology, Tsudanuma 275-0016, Japan

\begin{abstract}
Now we can use several temperature control modes, for example, isothermal run including stepwise heating and cooling, constant rate heating (or cooling), temperature control for sample thermal history, sample-controlled thermal analysis (SCTA or controlled-rate thermal analysis, CRTA), temperature jump, rate jump, temperature modulation, and repeated temperature scanning. Their advantages and drawbacks are reviewed with some illustrative examples, especially for application to kinetic analysis. The combined use of these varieties of temperature control mode is recommended by showing examples. Temperature modulation and repeated temperature scanning are discussed in comparison with temperaturemodulated differential scanning calorimetry (DSC), and common and analogous points are elucidated. In relation to this, the possibility of the imaginary part of the overall reaction rate constant in complex reactions is postulated. Finally, these modes are classified and tabulated from two viewpoints, and other possible modes are shown.
\end{abstract}

\section{INTRODUCTION}

For a recent few decades, we have observed remarkable development in thermal analysis [1], especially in new techniques by which chemical and structural changes in the sample can be directly observed. Thermal analysis by Fourier transform infrared spectroscopy is one example [2]. In other examples, X-ray diffraction is observed simultaneously during differential scanning calorimetry (DSC) [3], and volatilized products are analyzed by mass spectrometry as evolved gas analysis (EGA) together with thermogravimetry (TG) [4]. By these new microscopic techniques, we can directly observe chemical and physical changes in the sample. Thus, we can learn what is going on in the sample. However, observing bulk physical property changes by classic thermal analysis (e.g., enthalpy changes by DSC, mass changes by TG, and dimension changes by thermodilatometry), we can only learn something is going on in the sample, but we cannot learn what is going on. Comparing the classic thermal analysis with the new microscopic thermal analysis, great progress in thermal analysis is clearly realized.

Another aspect of the development in thermal analysis is diversification of temperature control modes, and nowadays we have the following modes of temperature control;

- $\quad$ isothermal run, including stepwise heating and cooling

- constant rate heating (or cooling)

- $\quad$ temperature control for sample thermal history [5]

- $\quad$ sample-controlled thermal analysis (SCTA or controlled-rate thermal analysis: CRTA) [6]

- $\quad$ temperature jump [7] and rate jump [8]

- temperature modulation [9-11]

- $\quad$ repeated temperature scanning [12]

\footnotetext{
*Lecture presented at the $12^{\text {th }}$ International Congress on Thermal Analysis and Calorimetry, Copenhagen, Denmark, 14-18 August 2000.

†E-mail: ozawat@pf.it-chiba.ac.jp.
} 
The last two are different in data processing. In the temperature-modulated TG oscillating change of the physical property we observe is analyzed by Fourier analysis, and the amplitude of the oscillating rate of conversion is compared with that of temperature modulation [11], while equivalent isothermal curves of the conversion versus its rate are extracted in the latter. Details will be described below.

Each mode has its suitable applications, and the advantages and drawbacks are discussed in this paper by using illustrative applications, mainly in applications to kinetics analysis of polymer decomposition. Appropriate choice of these modes and combined use should be made. To make full use of these modes, methods for kinetic analysis is also examined, and Friedman-Ozawa plot $[13,14]$ is concluded to be the most suitable because of the most wide applicability. It is also postulated that an overall rate constant may have an imaginary part due to transient change to steady state, and it would be observed as phase shift in tm-TG (or tm-DSC) and delayed change in TG (or nonmodulated DSC) by repeated temperature scanning. These points are reviewed in this paper.

The third mode of temperature control is somewhat different from the other. To elucidate the nature of an observed physical transition, researchers used to observe the effect of heat treatment of the sample on the transition. One typical example is described in ref. 5, in which differential thermal analysis (DTA) was used in combination with an adiabatic calorimeter, and the effect of annealing was first observed by DTA prior to the calorimetry. In another example, the effect of heat treatment on polypropylene crystallization was observed $[15,16]$. In this mode of temperature control, a thermal analysis instrument, such as DSC and DTA, is used as a tool for sample heat treatment to give a desired thermal history to the sample, as well as a tool for observation. In TG, similar heat treatment is also made within a thermobalance, for instance, to completely dry the sample prior to the thermal decomposition to diminish the effect due to residual water [17]. Since this mode of temperature control is different from the other modes, it will not be discussed any more in this paper.

\section{ISOTHERMAL RUN}

Isothermal run is an old method utilized for many decades to observe reactions, and its results are very simple for us to kinetically analyze the data, but it takes a long time when we observe reactions and their temperature dependence. This is clearly shown in one example in the next section.

However, stepwise heating and cooling, which is one of the modifications of isothermal observation, is suitable to observe equilibrium as shown in an example. Figure 1 shows TG results for one of

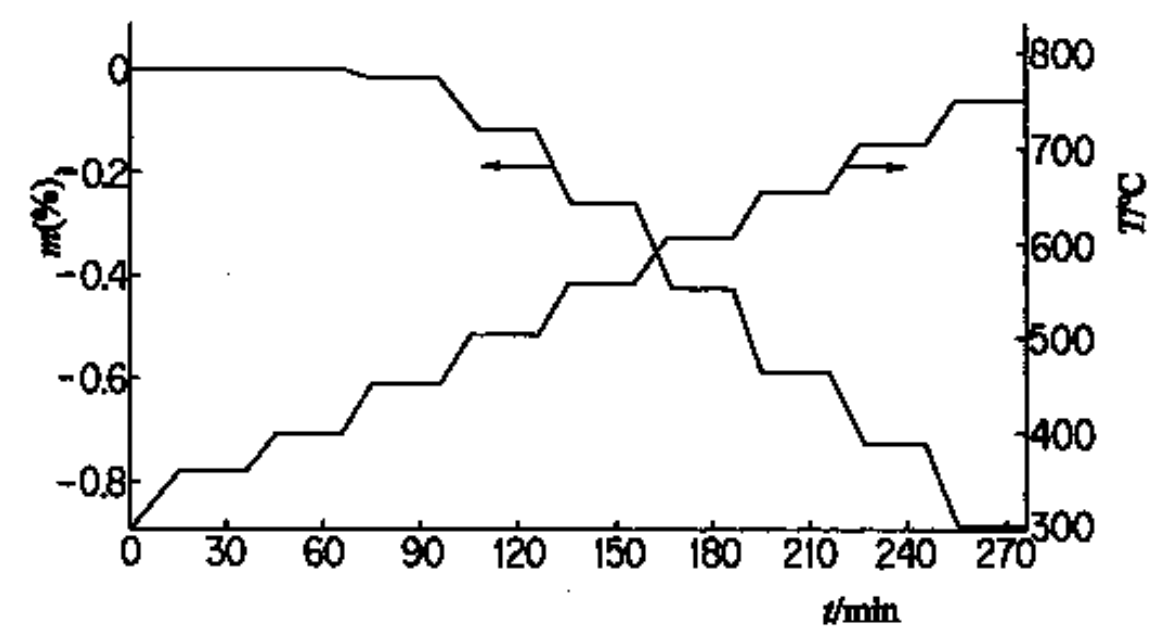

Fig. 1 TG curve of $\mathrm{YBa}_{2} \mathrm{Cu}_{3} \mathrm{O}_{7-\delta}$ by stepwise heating and cooling [18]. The symbols $m, T$, and $t$ are respectively the mass gain, the temperature, and the time. 
the oxide superconductors, $\mathrm{YBa}_{2} \mathrm{Cu}_{3} \mathrm{O}_{7-\delta}$, in ambient atmosphere by stepwise heating and then cooling [18]. Final mass at each step is plotted against the temperature (Fig. 2). Because the mass observed in heating is in good agreement with that in cooling, it can be concluded that the measured mass is the mass in equilibrium, and the nonstoichiometry is elucidated. This was a very important discovery, because the nonstoichiometry has much influence on the superconductivity. These results clearly illustrate the advantage of isothermal run for observing temperature dependence of properties in equilibrium. Similar stepwise heating was also made to obtain the relationship of molten fraction with the temperature for purity determination [19].

The stepwise heating and cooling could be applied to observe the temperature dependence of steady state. Furthermore, if the change in reacting species is negligible, it can also be applied to measurement of temperature dependence of reaction rate and hence the activation energy. This is used practically for thermal endurance evaluation of polymeric insulating materials [20].

\section{CONSTANT RATE HEATING}

The advantage of observation under constant rate heating over isothermal run can be shown by one typical example. When we observe thermal decomposition of poly(methyl methacrylate) under high vacuum by evolved gas analysis (EGA) with a mass spectrometer, we can get results shown in Fig. 3 [21]. As clearly seen in Fig. 3, volatilization by the thermal decomposition of this material proceeds as a four-step process. However, the product in each step is all monomer, and other products were scarcely detected, so that the four steps of the thermal decomposition are all depolymerization (i.e., unzipping of monomer from the radical end). Observing the dependence of the amount of volatilized monomer upon the initial degree of polymerization and atmosphere in the polymerization, we could conclude the following mechanism of the four-step unzipping process [21]. The first unzipping step starts at weak bonds, such as copolymerized oxygen. The second and third unzipping steps are initiated at the polymer ends. There can be three types of the polymer ends (i.e., the initiator radical end, the saturated end, and the unsaturated end by disproportionation in the annihilation of polymerizing radicals). But we can-

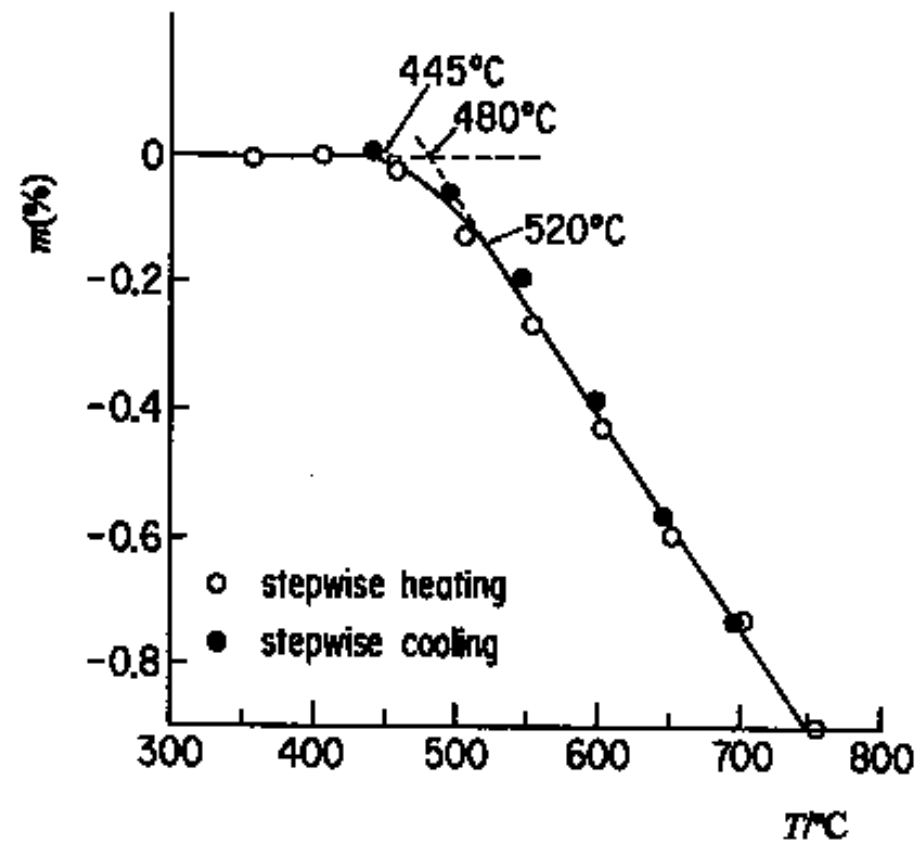

Fig. 2 Equilibrium mass change of $\mathrm{YBa}_{2} \mathrm{Cu}_{3} \mathrm{O}_{7-\delta}$ observed by stepwise heating and cooling [18].

(C) 2000 IUPAC, Pure and Applied Chemistry 72, 2083-2099 
not recognize which of these three types of the polymer ends are the causes for these two unzipping processes initiated at the polymer end. The fourth and final unzipping step starts by random scission in the polymer main chain. Each unzipping step is terminated with a relatively short kinetic chain length by radical recombination. These are overall thermal decomposition behavior of poly(methyl methacrylate) under high vacuum.

Imagining that we observed this process isothermally, as was done in the beginning of the polymer decomposition study, we can realize great advantages of constant rate heating over an isothermal run. When we observe the process isothermally at a relatively low temperature, we can observe the firststep reaction or the second-step reaction easily, but it is very hard to observe the third and the fourth steps, because they take a very long time and the rates of volatilization are very low. At a high temperature, the first and the second reactions proceed at a high rate, so that they would be completed in the time interval needed to heat up the sample to a desired isothermal temperature. Therefore, if we observe this process isothermally, we need a very long time to do numerous runs, otherwise it is very hard to reach a comprehensive, overall understanding of the process. Although kinetic analysis of data obtained by constant rate heating is somewhat complicated [17], this example clearly shows us the advantage of constant rate heating.

\section{SAMPLE-CONTROLLED THERMAL ANALYSIS}

One of advantages of constant rate heating is shown above, but its disadvantage is illustrated in the next example. When we observe the thermal decomposition of polyimide film in an ambient atmosphere by simultaneous TG-DTA at a constant rate, we get the results as shown in Fig. 4 [22]. It is clearly shown that the thermal decomposition of this material proceeds by two-step mass loss, and when we see the DTA curve, it is much clearer because we can see two exothermic peaks. Even if we change the rate of heating, the nature of the two-step decomposition essentially does not change. However, when we observe the same decomposition process in the same atmosphere by SCTA, namely, sample-controlled TG (SCTG or CRTG), the results we obtained are quite different, as shown in Fig. 5 [22]. Because the temperature changes smoothly and does not change discontinuously to keep a constant mass loss rate, we can conclude that the decomposition proceeds by a single-step process.

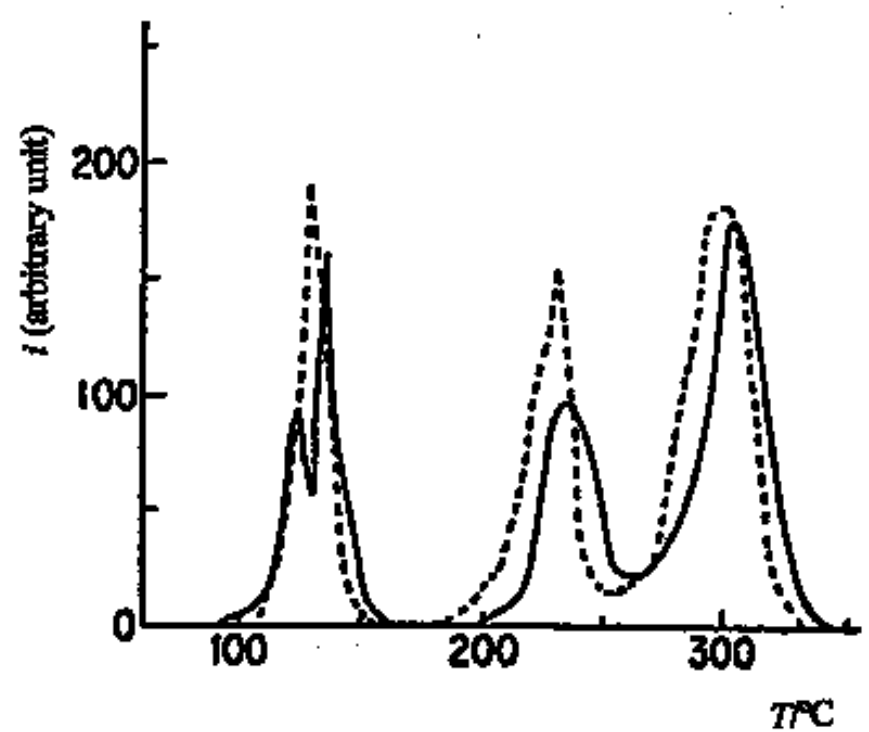

Fig. 3 EGA curves of thermal decomposition of two samples of poly(methyl methacrylate) under high vacuum [20]. The symbol $i$ is the ion current. 


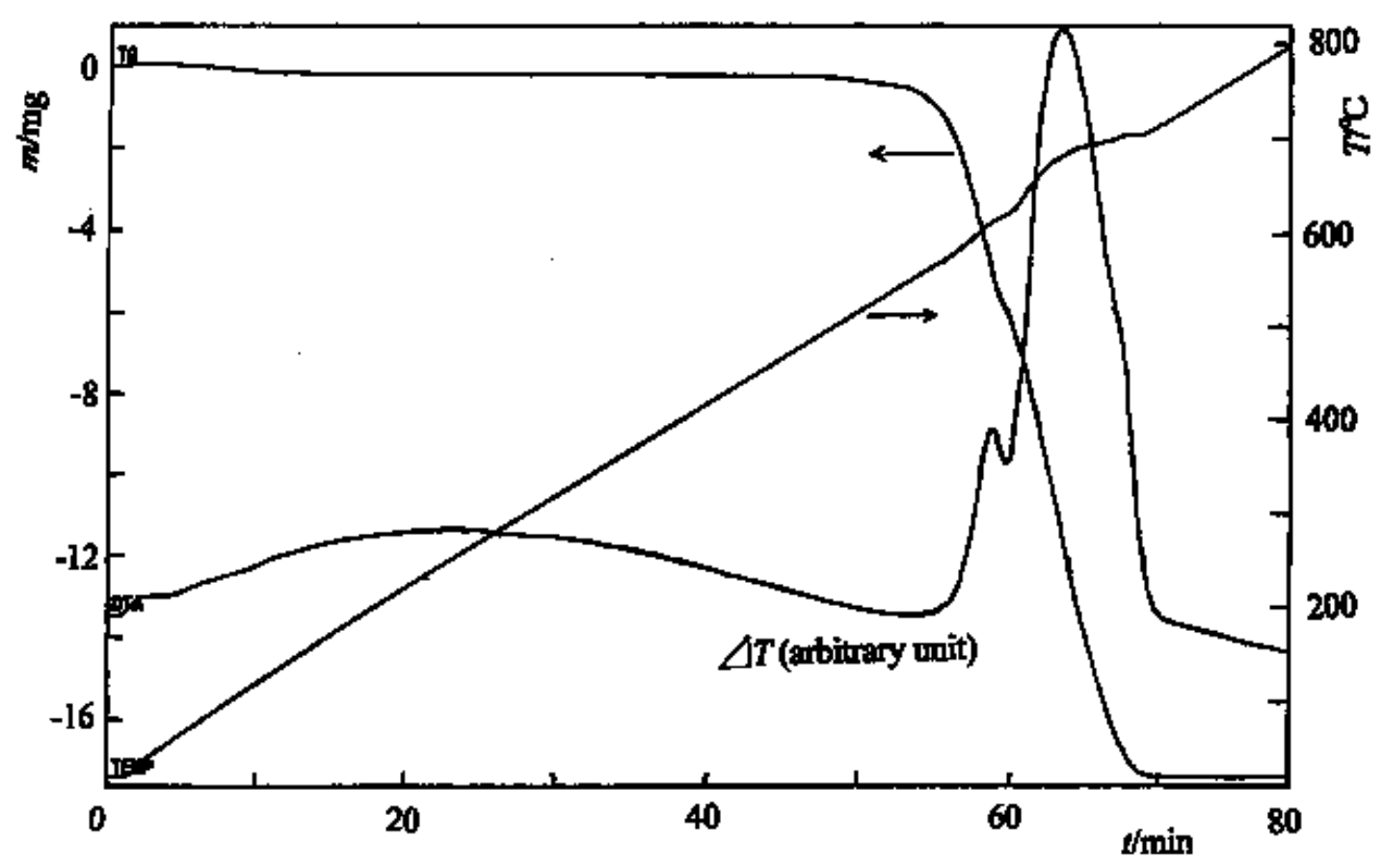

Fig. 4 TG-DTA curve of thermal decomposition of polyimide film in air flow by constant rate heating at $10^{\circ} \mathrm{C} / \mathrm{min}[21]$. The temperature difference $\triangle T$ by DTA is shown in an arbitrary unit.

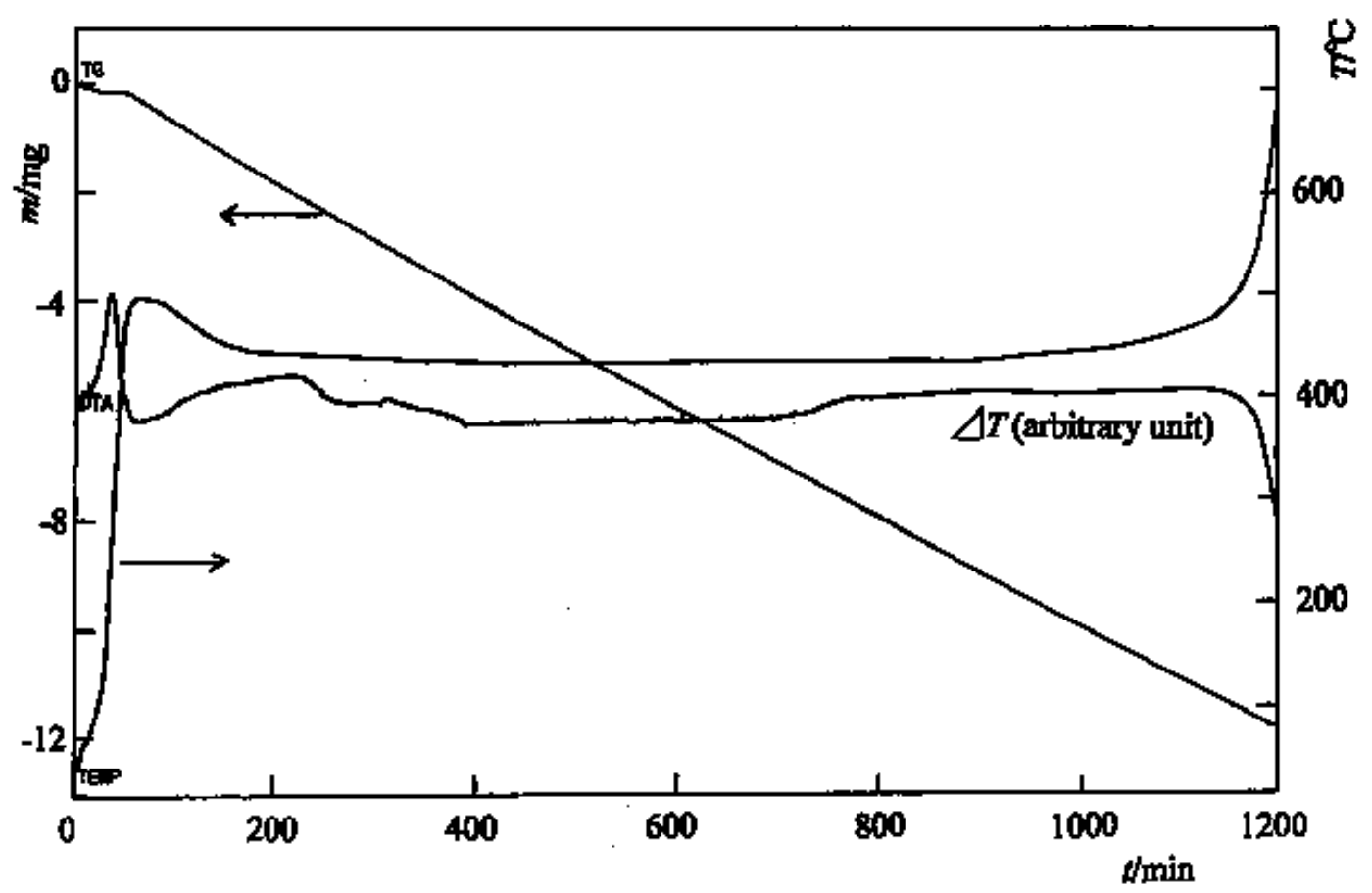

Fig. 5 SCTG curve of polyimide film in air flow obtained at mass loss rate of $0.06 \% / \mathrm{min}^{-1}$ [21]. The temperature difference by DTA is also shown.

(C) 2000 IUPAC, Pure and Applied Chemistry 72, 2083-2099 
To examine these contradictory results, kinetic analysis was applied to both data by constant rate heating and by SCTG. For this purpose, Friedman-Ozawa plot $[13,14]$ is only a reliable isoconversion method for estimating the activation energy, because this plot can be applied to data obtained by any temperature change. In the other isoconversion methods (i.e., Ozawa-Flynn-Wall plot $[17,23]$ and Kissinger-Akahira-Sunose plot $[24,25]$ ), however, the constant rate heating is one of the prerequisites. Therefore, they cannot be applied to data by the other temperature control modes. The results are shown in Figs. 6a and 6b, in which data obtained by isothermal runs are also plotted. Linear plots are obtained for data in a temperature range below about $500{ }^{\circ} \mathrm{C}$, but data above this temperature tend to deviate from the linear plots. Thus, this fact suggests that the decomposition mechanism changes in this temperature range.

To confirm this speculation, isothermal observations were done in this temperature range, and the results are reproduced in Fig. 7. It was confirmed that the thermal decomposition below $500{ }^{\circ} \mathrm{C}$ proceeds by a single step (Fig. 7a), while it is a two-step process above $550{ }^{\circ} \mathrm{C}$ (Fig. 7b). By observing this process by SCTG at a higher mass loss rate, we can observe two-step decomposition in a higher temperature range.
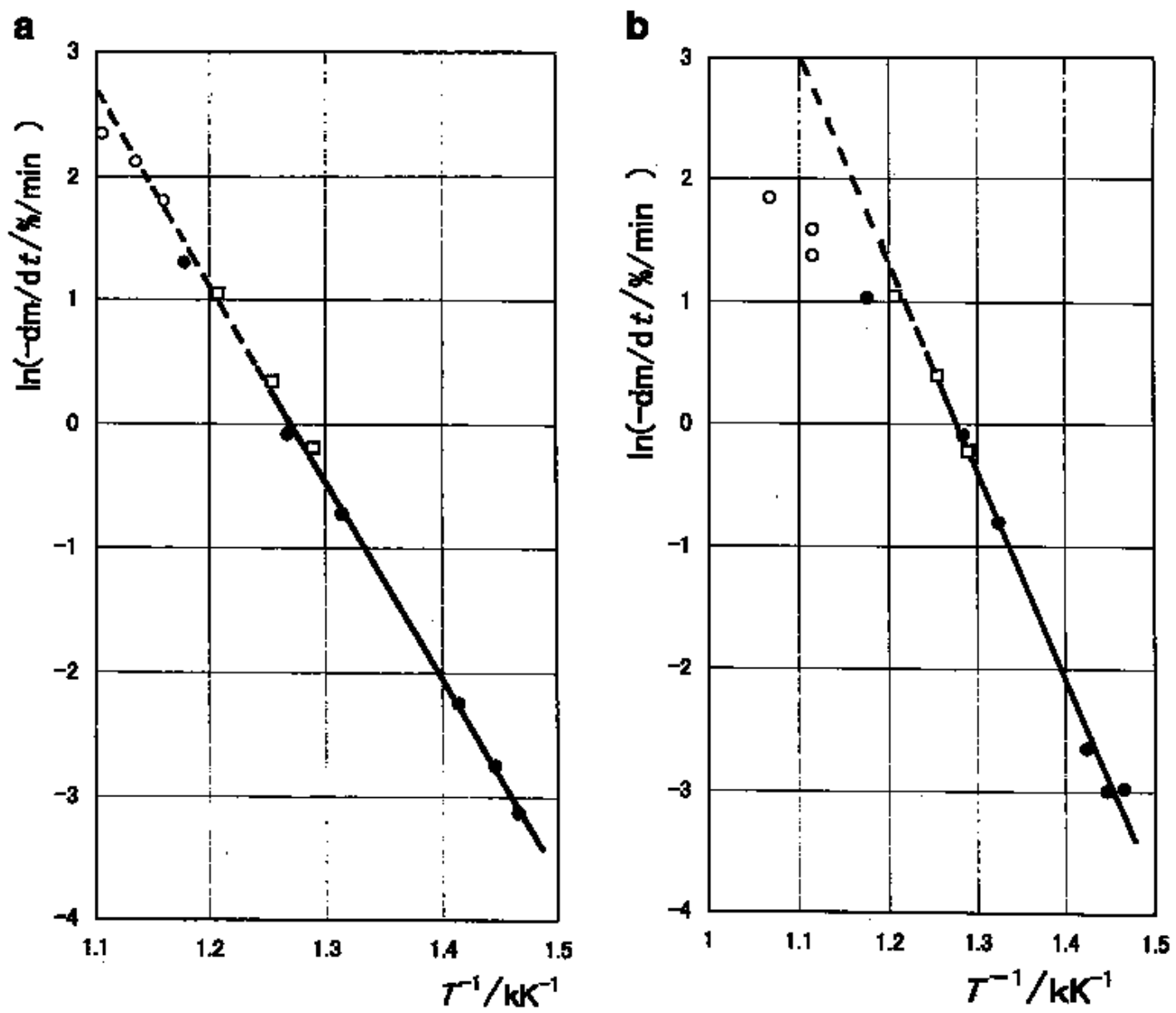

Fig. 6 Friedman-Ozawa plot for thermal decomposition of polyimide film [21]. The symbols $\bigcirc$, •, and $\square$, are respectively data by constant rate heating, SCTG, and isothermal run. Mass loss conversion of 25\% (a) and $35 \%$ (b). 

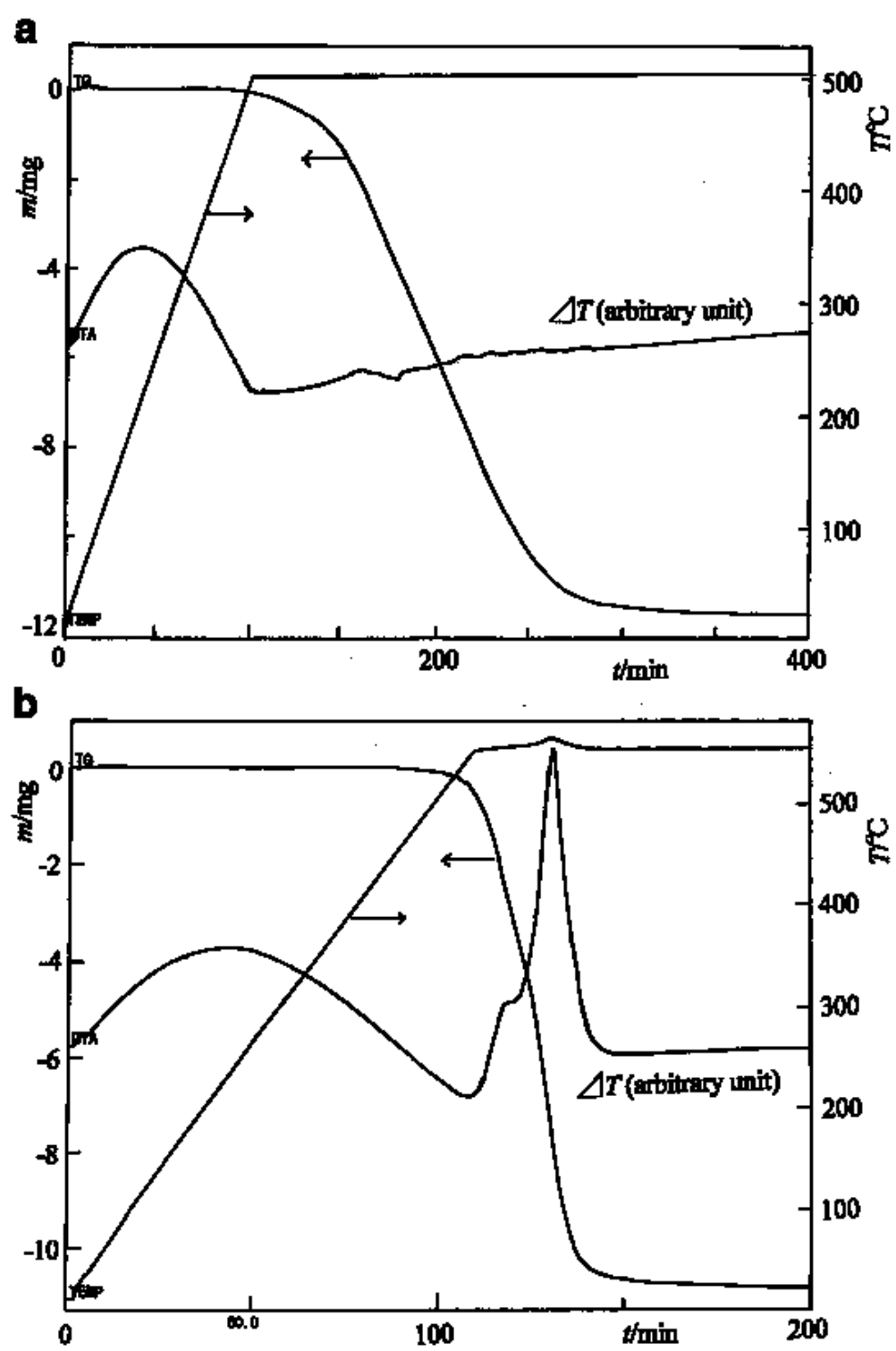

Fig. 7 Isothermal TG-DTA curves of polyimide film in air flow obtained at $500{ }^{\circ} \mathrm{C}$ (a) and $550{ }^{\circ} \mathrm{C}$ (b) [21].

The thermal decomposition behavior of this material is, thus, shown schematically in Fig. 8 [22]. There are competing two-reaction processes. One is the single-step mass loss and is predominant in a low temperature range. The other is the two-step mass loss and is predominant in a high temperature range. By isothermal run we observe either of these two processes, as shown with arrows in the figure. By constant rate heating we observe both; first the low-temperature predominant process is observed, but later the high-temperature process becomes predominant, as the temperature increases. When we observe the decomposition by controlling the mass loss rate at a relatively low rate by SCTG, we can mainly see the low-temperature predominant process. However, when we observe it at high rate of mass loss by SCTG, the high-temperature predominant process is observable. Thus, what we see by thermal analysis is changed by the temperature control mode we applied. 


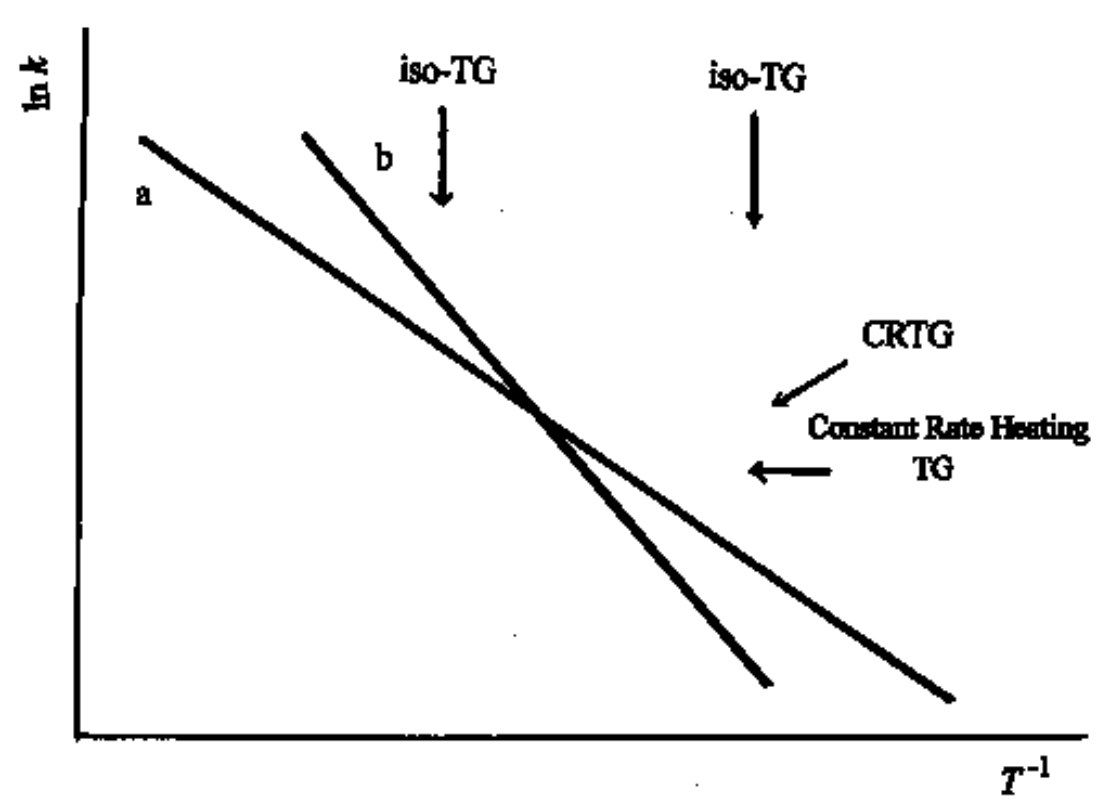

Fig. 8 Schematic expression for temperature dependence of reaction rate constants, $k$, for two competing reactions [21].

The usefulness of SCTA has been clearly elucidated for consecutive reactions of inorganic substances, especially for stepwise dehydration of crystalline water, and it is also shown for organic substances in differentiated evaporation of crystalline water from free water. Thus, the high resolution of SCTA has been well recognized. However, another usefulness of SCTA is shown above, and in this application an advantage of SCTA was revealed that the reaction proceeds at a constant desired rate in an automatically searched appropriate temperature range. Thus, this illustration also suggests to us that we can investigate real thermal behavior only by a combined use of various temperature control modes.

Another advantage of SCTG was postulated for polymer decomposition [26]. When thermal decomposition of poly(butylene terephthalate) under nitrogen flow was observed by constant rate heating modes, and the activation energy was estimated by the Ozawa-Flynn-Wall plot, the estimated activation energy is dependent apparently on the sample mass [27]. However, when it was observed at various constant mass loss rates by SCTG and the data were analyzed by Friedman-Ozawa plot, the activation energy estimated for a large sample mass is in good agreement with that estimated for a small mass sample by constant rate heating (see Fig. 9) [26]. Thus, it seemed that the apparent sample mass effect was not detected in SCTG results. Because the mass loss rate is controlled at a given rate in SCTG, volatilization of the products and, hence, the product partial pressure above the sample are also roughly controlled, and this controlled atmosphere seems to be a cause for the activation energy independent of the sample mass.

\section{KINETIC ANALYSIS METHODS}

It seems worthwhile to discuss the kinetic analysis methods in relation to the temperature control modes. It has been recognized that isoconversion methods, such as the Ozawa-Flynn-Wall plot [17,23], the Kissinger-Akahira-Sunose plot [24,25], and the Friedman-Ozawa plot [13,14], are the most reliable methods for estimating the activation energy using thermoanalytical data [28], because these three plots are all so-called "model-free".

As clearly seen in the above, however, the Friedman-Ozawa plot has an advantage over the Ozawa-Flynn-Wall plot and the Kissinger-Akahira-Sunose plot. In other words, the Friedman-Ozawa 


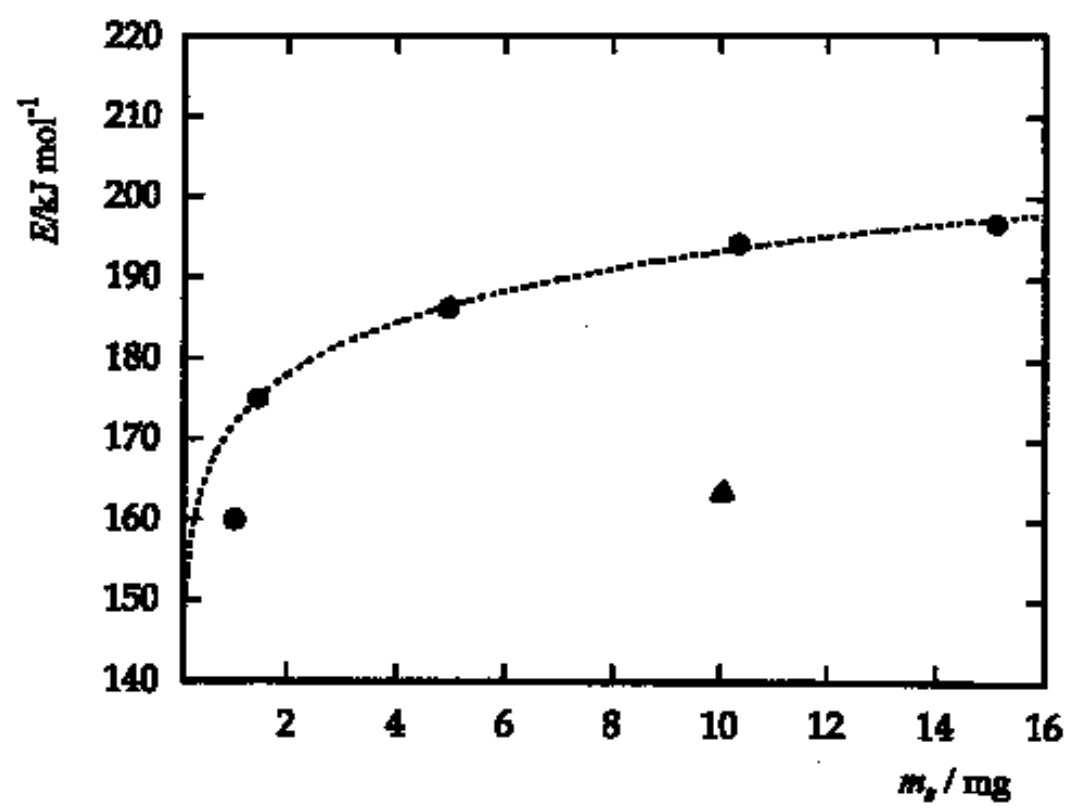

Fig. 9 Sample mass $\left(m_{s}\right)$ dependence of activation energy $(E)$ obtained by TG of poly(butylene terephthalate) [26]. The symbols, $\bigcirc$ and $\boldsymbol{\Delta}$, are respectively data by constant rate TG and SCTG.

plot is a single method that is applicable to data obtained by the combined use of various temperature control modes. Because the combined use of different temperature control modes is a powerful means to get overall thermal decomposition behavior, this point is very important for kinetic investigation of materials, especially organic materials.

When these three plots were devised, a computer was not installed with a thermal analysis instrument, so that it is hard to get the rate of conversion for integral-type thermal analysis, such as TG. At the same time, constant rate heating was mainly used for almost all thermal analysis. Under these historical circumstances, the three plots were proposed, and the Ozawa-Flynn-Wall plot and the Kissinger-Akahira-Sunose plot have been widely applied to data by constant rate heating, because it needs only the conversion and the temperature, not the rate of conversion. Friedman proposed his plot in 1964 [13], but it was not so easily applicable, because the rate of conversion is needed. Furthermore, Friedman applied his plot only to $n$-th order reaction, so that applicability of his initial plot was thought to be limited. Later, the present author found it is applicable to other processes as widely as the other two plots (expanded Friedman plot or Friedman-Ozawa plot) [14]. As computerization promoted, the situation has changed and Friedman-Ozawa plot became an easily applicable and reliable plot for estimating the activation energy. The other two plots are reliable, but they have a historical limit, because their applicability is limited.

It should also be noted that the reduced time or the generalized time is very useful to learn the mechanism function of the process by deriving experimental master curves using the generalized time or the reduced time $[17,29,30]$. In a course of this derivation, we can also examine the applicability of the method to the process under investigation [17,29]. The concept of the reduced time is independently applied to all data by any temperature control mode, although the calculation of the reduced time for constant rate heating is not so difficult as for other modes.

\section{TEMPERATURE JUMP AND RATE JUMP}

These jump methods were devised to estimate the activation energy. As is illustrated in Fig. 10, when we abruptly change the temperature as shown at the bottom, this jump in the temperature is reflected in 


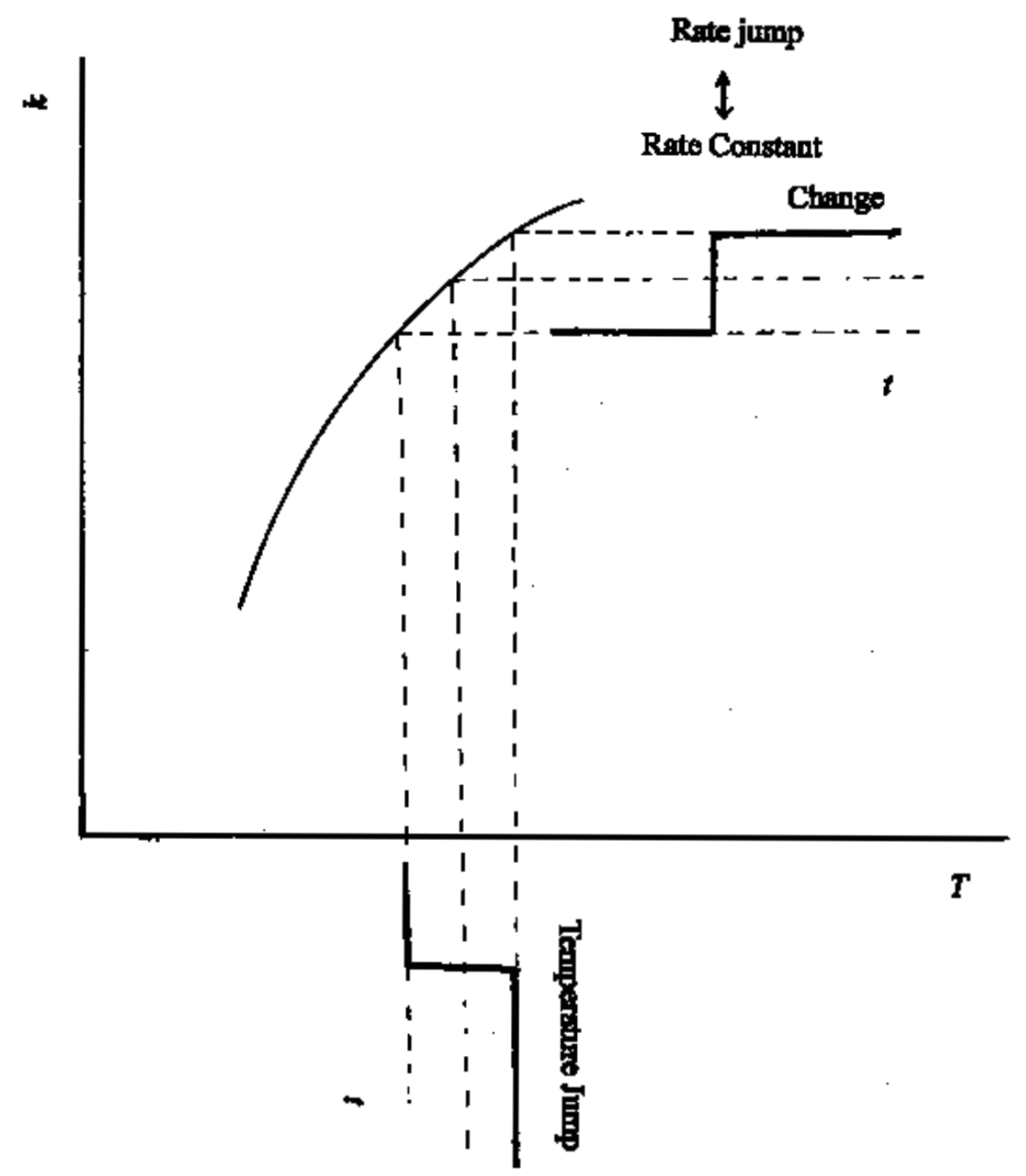

Fig. 10 Schematic explanation of jump methods.

a corresponding jump in the rate constant, and hence it appears as a jump in the conversion rate we are observing [7]. In reverse, when we change abruptly the conversion rate in SCTA, it is reflected in the temperature jump [8]. Therefore, we can compare the rate constants of the two temperatures at the rate jump or the temperature jump, because the rate is proportional to the rate constant at the jump, namely at a given conversion. This is based on the same principle with Friedman-Ozawa plot, so that it is widely applicable. These jump methods, therefore, are the most direct method to estimate the activation energy. In practical experiment, the temperature and the conversion rate do not change so abruptly as in the figure due to the thermal response of the system, and the rate or the temperature extrapolated to the given conversion at the jump is used for the evaluation of the activation energy.

These jump methods seem to be useful for kinetic analysis of parallel competitive reactions. For these reactions a method is proposed, and in this method the volatilization rates of products by each competing reaction are measured and compared with each other at a given amount of residual reacting species [31]. This is also an expansion of Friedman-Ozawa plot. However, one of the difficulties of this method is to specify the point of the given amount of residual reacting species for different runs, if we do not have a tool for directly measuring the amount of residual reacting species. Otherwise, we should know exact chemical stoichiometry of all products, because the relative amount of each product is 
dependent on the path or the trajectory of the process [32], and thus we can estimate the amount of residual reacting species from the amount of all volatilized products. By the jumps in the temperature or the rate of a certain volatile product, on the other hand, we can compare the volatilization rates of products by each competing reaction at a given amount of residual reacting species at different temperatures. Therefore, we can observe the difference in the temperature dependence of the rate constant among the competing reactions, and it is the first step to elucidate the mechanism. A similar advantage of the jump methods seems expected also for consecutive reactions.

\section{TEMPERATURE MODULATION}

Temperature modulation was first introduced into thermal analysis as temperature-modulated DSC [9,10], and since its publication in 1992 various researches have been done to investigate complex response of the sample under temperature modulation. In this course of progress, temperature modulation was applied to other techniques, and temperature-modulated TG (tm-TG) was proposed [11]. The sinusoidal temperature modulation causes oscillation of the rate constant and hence oscillation of the rate of mass change, as illustrated in Fig. 11.

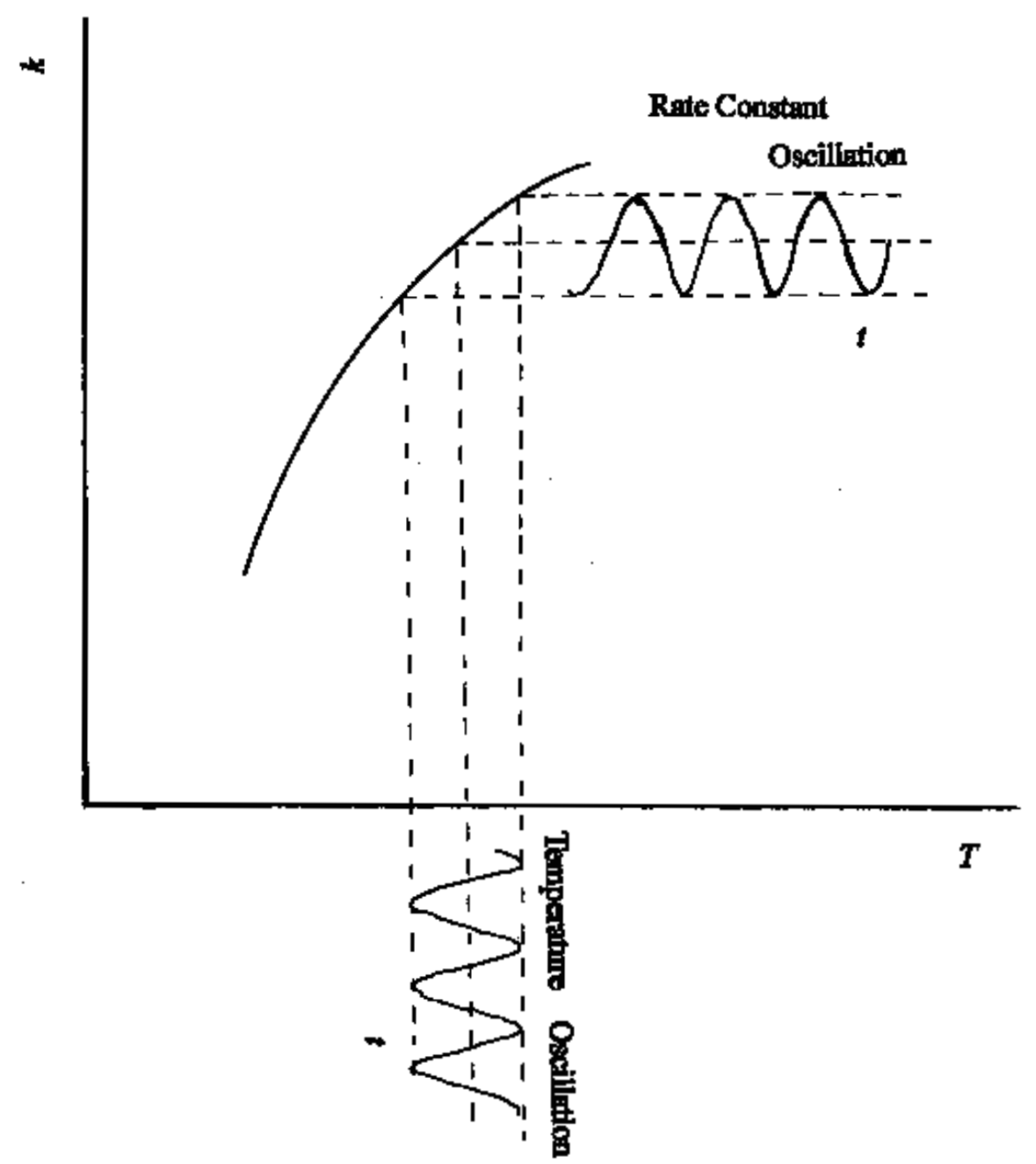

Fig. 11 Schematic explanation of temperature modulation method.

(C) 2000 IUPAC, Pure and Applied Chemistry 72, 2083-2099 
In tm-TG, the oscillating rate of mass loss by the temperature modulation is observed together with mass loss. In some cases, the temperature modulation is superimposed on the underlying constant rate heating, and in other cases of quasi-isothermal measurement the temperature is modulated around the underlying constant temperature. One of the examples of tm-TG curves is reproduced in Fig. 12 [11]. In both ways of tm-TG the amplitude of the oscillating rate of mass loss is compared with the amplitude of temperature modulation, and the activation energy is estimated in real time by this comparison.

There are a few points to be noted [12]. The first is nonlinear dependence of the rate constant on the temperature. Due to this nonlinearity, the sinusoidal temperature modulation is nonlinearly reflected in the oscillating rate, and the rate oscillation becomes deformed. In other words, the accelerating effect in the temperature range above the average temperature is lower than the deceleralating effect in the lower temperature range, so that the change in the rate constant becomes different from the sinusoidal change. Because the amplitude of the rate oscillation is calculated by Fourier analysis based on the linearity, the linearity is one of prerequisites for this method to estimate the activation energy. Therefore, the essential nonlinear effect should be negligible, so that the amplitude of the temperature modulation should be low, for instance less than $\pm 10^{\circ} \mathrm{C}$, but it depends on the temperature and the activation energy.

As mentioned above, the temperature modulation has been applied to various thermal analyses. Although it has not yet been applied to EGA, it would become a useful and interesting technique especially for parallel competitive reactions because of the above reasons. By comparing the amplitudes of oscillating volatilization rates of various products, we could learn the difference in the activation energy and the number of involved reactions.

\section{REPEATED TEMPERATURE SCANNING}

This temperature control mode is similar to temperature modulation but different in the kinetic method [12]. In this temperature control mode, the sample is repeatedly heated and cooled in a desired and defined temperature range, and the triangular saw-teeth modulation is preferable to the other modulations, because the reduced time can be easily calculated for this modulation. Because the rate of con-

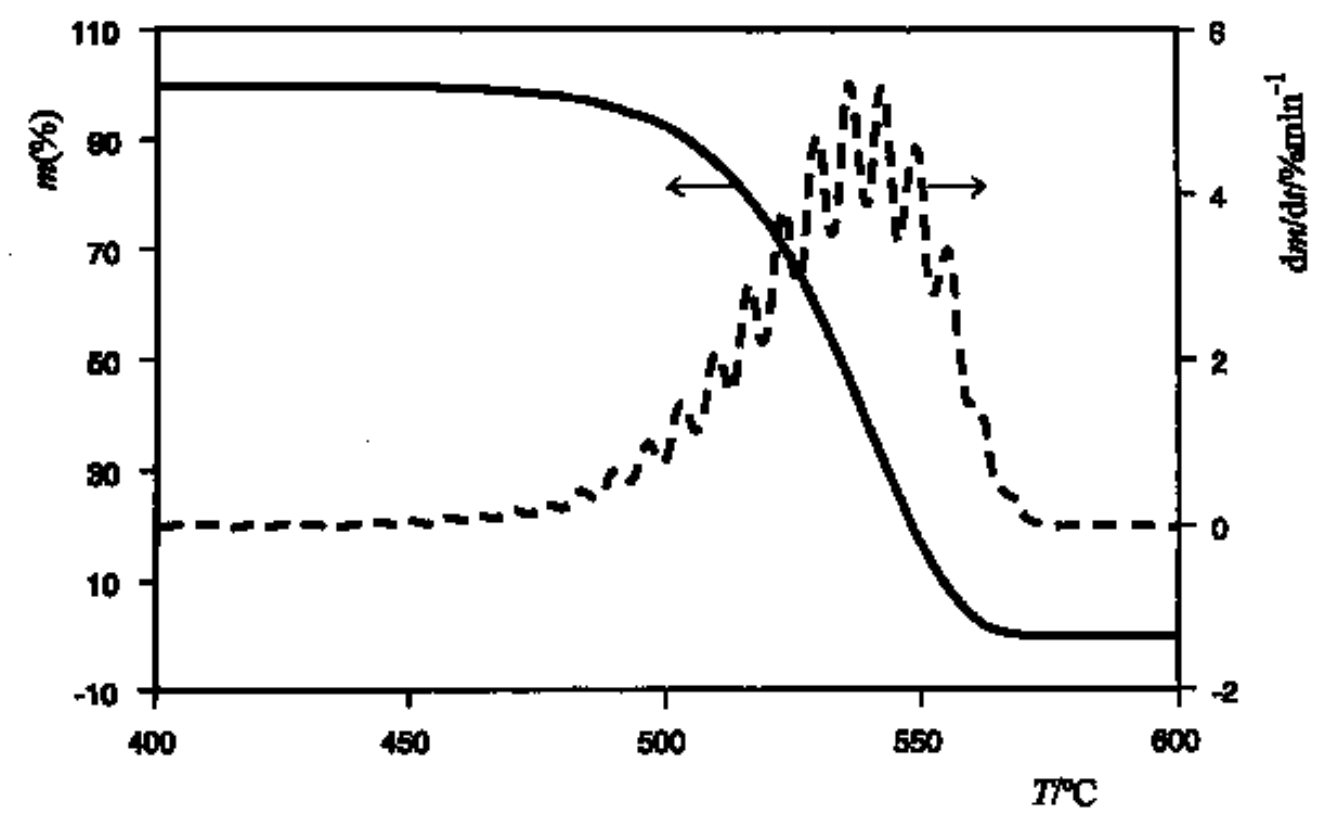

Fig. 12 Temperature-modulated TG curve of polytetrafluoroethylene [11]. 
version is the same at a given conversion and a given temperature in any temperature control modes, the relation between the rate of conversion and the conversion can be extracted at a given temperature from data obtained by this temperature mode. This relation is an equivalent isothermal curve of the rate of conversion versus the conversion, and it is equal to that obtained by isothermal run. When we make similar extraction at different temperatures, we can get equivalent isothermal curves at multiple different temperatures. Thus, it is the essence of the method that data by repeated temperature scanning are transformed to multiple equivalent isothermal curves.

This method was applied to thermal decomposition of poly(methyl methacrylate) of high molecular mass and poly(butylene terephthalate) in nitrogen flow [33]. A typical result is shown in Fig. 13. By extracting the conversion and the rate of conversion at given temperatures, we got multiple equivalent isothermal curves for poly(methyl methacrylate), and they are reproduced in Fig. 14. As described above, this material undergoes unzipping, and it is a first-order reaction, which is clearly seen in linear relations in Fig. 14. A slight difference was observed between the data obtained in the heating mode and those in the cooling mode. This is discussed below. Thus, the usefulness of this temperature control mode is clearly shown, and it is also very clear that it is time-saving.

For poly(butylene terephthalate), equivalent isothermal curves are shown in Fig. 15. As seen in this figure, we got the two curves for one temperature; the curves obtained in the heating mode are different from those in the cooling mode, although the arched form of curves is typical for random scission in the main chain of this polymer [26,27]. One of the plausible causes for this discrepancy seems to be temperature lag. The recorded temperature is not the sample temperature, but the temperature of the temperature sensor (the thermocouple), and there is a temperature gradient around the sample for heat flow. In this particular case in Fig. 15, the sample temperature changed with some lag from the recorded temperature, so that the sample temperature is lower by a certain degree than the recorded temperature in the heating mode and higher in the cooling mode. This effect is schematically shown in Fig. 16. It also exists in any temperature change, including the constant rate heating, but until now the reaction is usually not observed by cooling mode nor compared with heating mode, so that this effect has

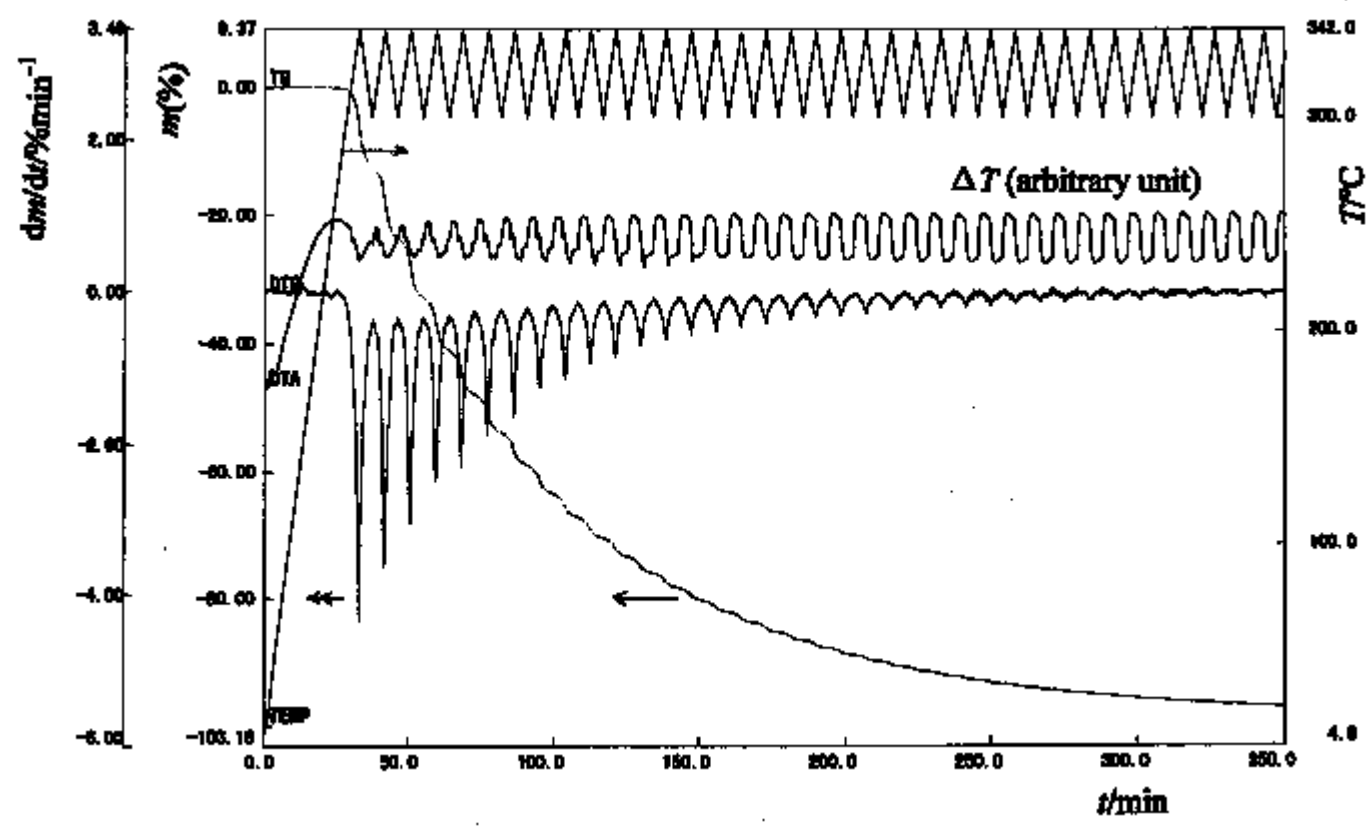

Fig. 13 T-DTA curve of poly(methyl methacrylate) by repeated temperature scanning. 


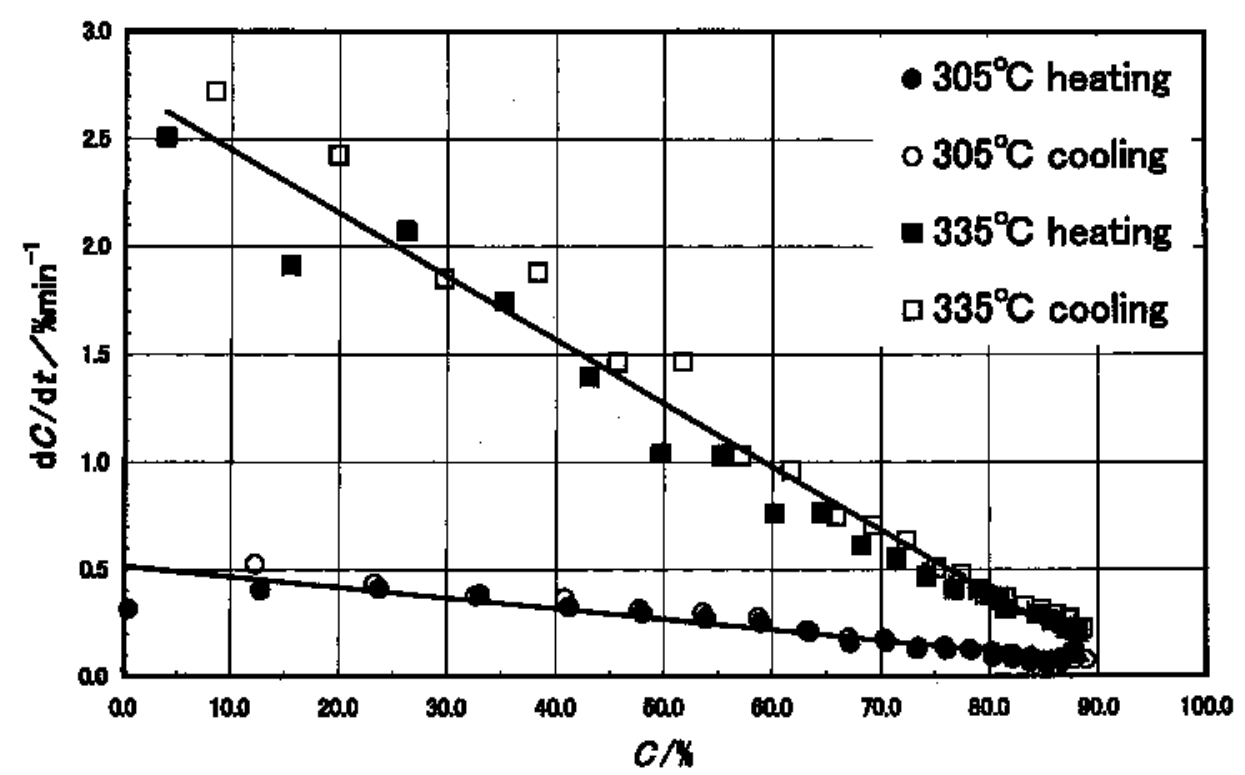

Fig. 14 Equivalent isothermal curves of conversion $(C)$ versus conversion rate extracted from TG curve of poly(methyl methacrylate) in Fig. 13.

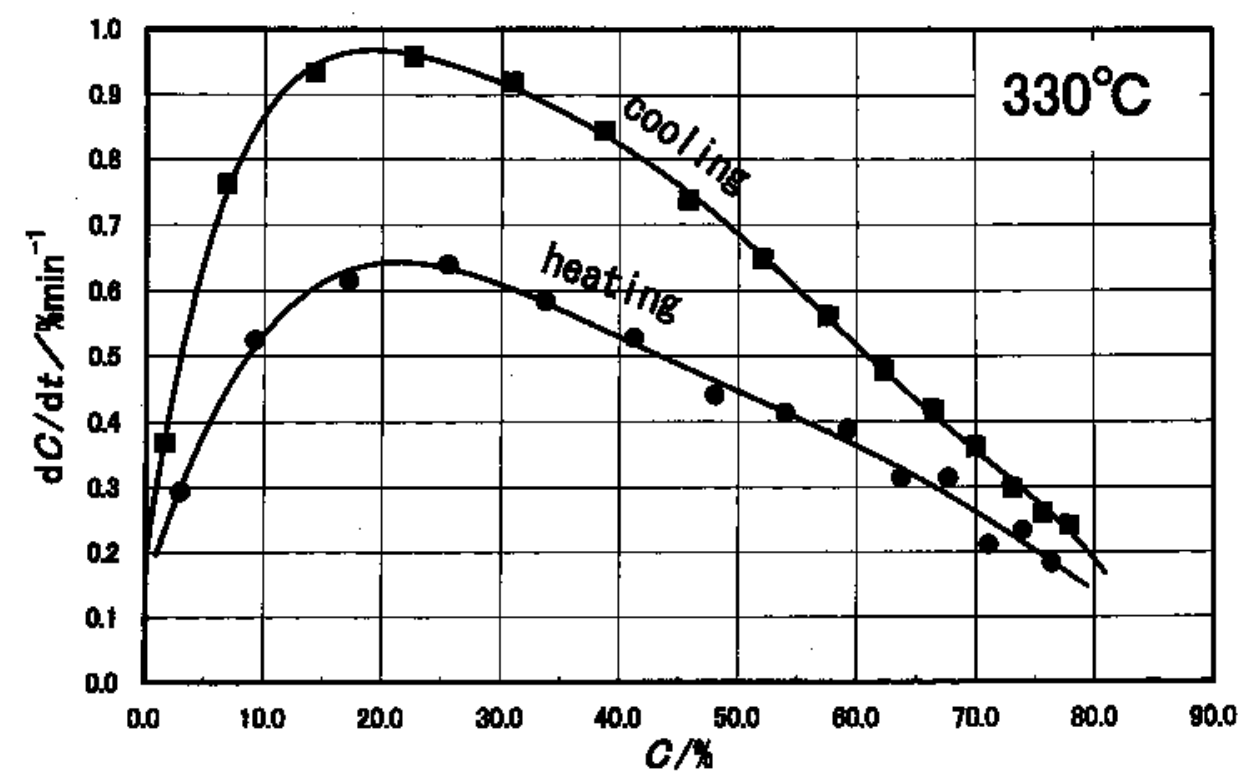

Fig. 15 Equivalent isothermal curves of conversion rate versus conversion for poly(butylene terephthalate) at $330{ }^{\circ} \mathrm{C}$.

not yet been noticed. It is also clearly shown that the temperature measured by TG should be corrected by using standard materials, such as magnetic standards [34]. The temperature difference between the heating mode and the cooling mode was also detected by using the magnetic standards [34].

This temperature lag seems to exist in tm-TG as a phase shift in the mass loss rate, although it has not yet been reported, and this exists in tm-DSC [35]. Furthermore, this effect is common in the technique by temperature modulation. When modulated temperature wave is propagating in the instrument 


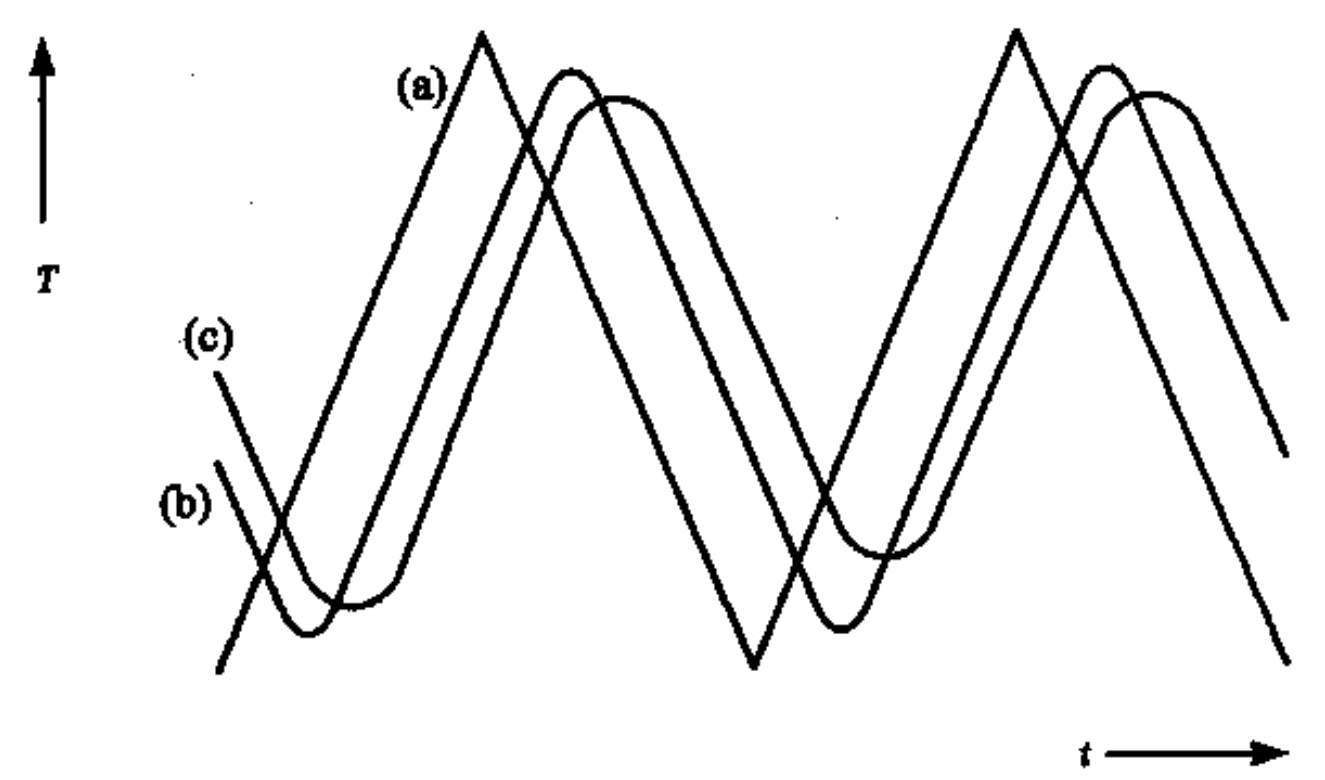

Fig. 16 Schematic drawing of recorded temperature (a), sample temperature (b), and rate change (c).

and in the sample by thermal conduction, the amplitude is always decreased and the phase is shifted, and thermal contacts among the sample, the cell, and the cell holder plate are other causes for this effect. The higher the frequency is, the larger the effect is. Therefore, there are the same and common problems between the repeated temperature scanning and the temperature modulation. The heat of reaction also disturbs the temperature control.

However, there might be another cause for the above discrepancy, and it is transient change to the steady state. One possible example is the unzipping of the monomer from the radical ends $[21,36]$. As described above, this process proceeds as a first-order reaction in a steady state in which the radical concentration is kept at a constant concentration, because the rate of annihilation of the radical by recombination becomes equal to that of the radical formation by chain scission. The rate of unzipping is proportional to this constant radical concentration, and this concentration is dependent on the temperature mainly because of the activation energy of the radical formation. In the repeated temperature scanning, the radical concentration changes as the temperature changes. If there is a certain lag in this change of the radical concentration, it becomes a cause for the delay in change in the rate of unzipping. Thus, there is a possibility that transient change to the isothermal rate might be detected as the delay in the temperature change of the rate in the heating and cooling mode, and it is also detected in retarded change of the rate in the temperature jump. In tm-TG it appears as the phase lag and the amplitude decrement. Thus, there might be an imaginary part in the overall rate constant of complex reactions.

This is analogous to imaginary part of heat capacity [35]. However, the imaginary part of heat capacity is caused by relaxation to the equilibrium, but the imaginary part of overall rate constant is different from the relaxation but the transient change to the steady state in the complex reaction. Both of these imaginary parts cause the decrease in the amplitude and the shift in the phase (or the delay in the rate change in the repeated temperature scanning and in the jump methods). Therefore, these changes are observed together with the similar effect by the temperature wave propagation (or the temperature lag), so that a problem to be solved is to separate the apparent effect by these instrumental and procedural causes from the imaginary part which seems to really exist. This is also a common problem between the repeated temperature scanning and the temperature modulation and also between tm-DSC and tm-TG. 
Table 1 Classification of temperature control modes.

\begin{tabular}{|c|c|c|c|c|}
\hline & & \multicolumn{3}{|c|}{ 9y-po-dCond } \\
\hline & & 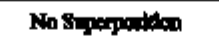 & Motulation & Rpoted So ming \\
\hline \multirow{3}{*}{$\begin{array}{l}\text { Undutytng } \\
\text { Coonion }\end{array}$} & Centert Thuporture & Loth-m-1 & Qintinghed & 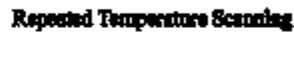 \\
\hline & 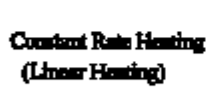 & 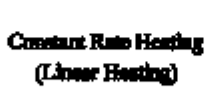 & Imor Heth: & Not Nomed \\
\hline & 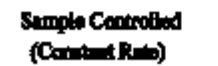 & $\frac{\operatorname{sct}}{(\operatorname{CrTA})}$ & PAMotiltan & 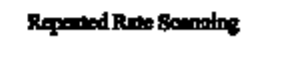 \\
\hline
\end{tabular}

\section{CLASSIFICATION AND OTHER MODES OF TEMPERATURE CONTROL}

The temperature control modes described above can be classified from two viewpoints, (i.e., the underlying control and the superposed control), as shown in Table 1. From this classification, other possible temperature control modes are revealed, as shown in the table, but some of them are not yet named properly.

The underlying control modes have common characteristics, which are independent of the superposed control modes. In the case of constant rate heating, the process can easily be observed up to its final stage by accelerating effect of the temperature increase. Similarly, we can also observe the final stage by the SCTA mode within the estimated time, because the rate of conversion is controlled. On the other hand, by the isothermal run, the conversion rate decreases and finally becomes so low that it cannot be measured because of low signal/noise ratio and we can hardly observe the change in the conversion per se.

The superposed control modes have also common characteristics. By the modes of the temperature jump, the rate jump, the temperature modulation, and the rate modulation, the activation energy is estimated easily by comparing the temperature jump versus the rate jump or the temperature amplitude versus the rate amplitude. However, they are not so suitable to elucidate the mechanism function as the others, and the amplitudes should not be so large, as mentioned above. In the temperature-scanning mode and the rate-scanning mode, the equivalent isothermal relations between the conversion and the conversion rate are directly extracted, so that the mechanism function can be elucidated easily.

\section{SUMMARY}

- The advantages and drawbacks of various temperature control modes are shown with some examples of application.

- A choice between these modes is required to make appropriate thermal analysis.

- Furthermore, the combined use of these modes is recommended to elucidate the whole thermal behavior of the sample, and it is important especially for organic substances, such as polymers, because the reaction mechanism might change by the temperature change.

- It was found by repeated temperature scanning that thermal decomposition observed in the cooling mode tends to differ from that observed in heating modes. It is caused by the difference between the recorded temperature and the sample temperature due to the thermal gradient around the sample for heat flow.

- However, there may be another cause, for example, a lag in the rate due to transient change to the steady state, which is also changing by the temperature change.

- $\quad$ These would be observed as amplitude decrement and phase angle shift in tm-TG. 
- $\quad$ These phenomena are common and analogous to those in tm-DSC.

- The various temperature control modes can be classified by the two viewpoints (i.e., the underlying control and the superposed control). Their characteristics or their advantages and drawbacks are described from these points of view.

\section{ACKNOWLEDGMENTS}

The author would like to express his appreciation for permission of figure reproduction from Prof. J. Simon, the editor of Journal of Thermal Analysis and Calorimetry, and also appreciation is extended to Elsevier Science Publishers, B. V. The author also thanks Dr. R. Blaine and Dr. T. Arii for their permission to reproducte figures in their papers.

\section{REFERENCES}

1. T. Ozawa. Thermochim. Acta 355, 35 (2000).

2. For example, H. Yoshida. J. Therm. Abal. 36, 4805 (1995).

3. For example, T. Arii, A. Kishi, Y. Kobayashi. Thermochim. Acta 325, 151 (1999).

4. For example, T. Ozawa, T. Arii, A. Kishi. Thermochim. Acta 352/353, 177 (2000).

5. For example, K. Ogasawara, M. Sorai, H. Suga. Mol. Cryst. Liq. Cryst. 71, 189 (1981).

6. J. Rouquerol. Thermochim. Acta 144, 209 (1989).

7. B. Dickens. J. Polym. Sci. - Polym. Chem. 20, 1065 (1982).

8. J. Rouquerol. J. Therm. Anal. 5, 203 (1973).

9. M. Reading, E. Elliot, V. L. Hill. J. Therm. Anal. 40, 949 (1993).

10. P. S. Gill, S. R. Sauerbrumn, M. Reading. J. Therm. Anal. 40, 931 (1993).

11. R. L. Blaine and B. K. Hahn. J. Therm. Anal. 54, 695 (1998).

12. T. Ozawa. Thermochim. Acta 356, 173 (2000).

13. H. L. Friedman. J. Polym. Sci. C6, 183 (1964).

14. T. Ozawa. J. Therm. Anal. 31, 547 (1986).

15. J. Varga. J. Therm. Anal. 31, 165 (1986).

16. J. Varga. J. Therm. Anal. 35, 1891 (1989).

17. T. Ozawa. Bull. Chem. Soc. Jpn. 38, 1881 (1965).

18. T. Ozawa, A. Negishi, Y. Takahashi, R. Sakamoto, H. Ihara. Thermochim. Acta 124, 147 (1988).

19. H. Staub and W. Perron. Anal. Chem. 46, 128 (1974).

20. T. Ozawa, K. Kaneko, T. Sunose. J. Therm. Anal. 47, 1105 (1996).

21. T. Ozawa, M. Kanazashi, R. Sakamoto. Thermochim. Acta 109, 285 (1986).

22. T. Ozawa. J. Therm. Anal. 59, 375 (2000).

23. J. H. Flynn and L. A. Wall. J. Polym. Sci. B4, 323 (1966).

24. H. E. Kissinger. Anal. Chem. 29, 1702 (1957).

25. T. Akahira and T. Sunose. Report Chiba Inst. Tech. [16] 22 (1971).

26. T. Arii, S. Ichihara, H. Nakagawa, N. Fujii. Thermochim. Acta 319, 139 (1998).

27. S. Ichihara, N. Nakagawa, Y. Tsukazawa. Kobunshi Rombunshu 51, 459 (1994).

28. T. Ozawa. Thermochim. Acta 203, 159 (1992).

29. T. Ozawa. J. Thermal. Anal. 2, 301 (1970).

30. T. Ozawa. Thermochim. Acta 100, 109 (1986).

31. T. Ozawa and K. Kanari. Thermochim. Acta 234, 41 (1994).

32. T. Ozawa. J. Thermal. Anal. 39, 1117 (1993).

33. A. Hishiki and T. Ozawa. Thermochim. Acta, To be submitted.

34. P. K. Gallagher. J. Thermal Anal. 49, 33 (1997).

35. T. Ozawa and K. Kanari. J. Thermal Anal. 59, 257 (2000).

36. H. H. G. Jellinek. Degradation of Vinyl Polymers, Academic Press, New York (1955).

(C) 2000 IUPAC, Pure and Applied Chemistry 72, 2083-2099 